\title{
Necessary and Sufficient conditions for Existence of Solutions of a Divergence-type Variational Problem
}

\section{José Matias}

Departamento de Matemática, Instituto Superior Técnico, Av. Rovisco Pais, 1, 1049-001 Lisboa, Portugal

E-mail address: jmatias@math.ist.utl.pt

Abstract. We look for necessary and sufficient conditions for the existence of solutions of the minimization problem

$$
(P) \quad \inf \left\{\int_{\Omega} f(P D u(x)) d x: u \in u_{\zeta_{0}}+W_{0}^{m, \infty}\left(\Omega ; \mathbb{R}^{N}\right)\right\}
$$

where $P D$ is a particular type of differential operator of order $m$, (which we identify as of divergence type) and the boundary data $u_{\zeta_{0}}$ satisfes $P D u_{\zeta_{0}}(x)=\zeta_{0}$ for $\zeta_{0} \in \mathbb{R}$ given.

\section{Introduction}

The search for minimizers of

$$
\inf \left\{\int_{\Omega} f(\nabla u(x)) d x: u \in u_{0}+W_{0}^{1, \infty}(\Omega)\right\}
$$

when the integrand function $f$ is non convex, has been undertaken extensively (see, for example, [3], [4], [8], [9] and the references therein). Dacorogna and Marcellini ([8]) showed that a necessary condition for existence of solutions to this problem is that the convex envelope of $f, f^{* *}$, is globally affine.

This work follows closely [1] where the problem is treated in the general setting of differential forms and [2] where the problem was treated in the case of the curl operator.

The research of José Matias was partially supported by FCT (Portugal) through the Program POCI/2010/FEDER and through the Project POCI /FEDER/MAT/55745/2004.. 
However, the (simpler) case of divergence was not explicitely treated. In this work, we deal with the problem for a particular type of differential operators, that namely comprise the divergence operator.

In particular, combining this work with the results of [1], [7] and [2], for $d u$ a differential form of order $k$ in $\mathbb{R}^{3},(0 \leq k \leq 2)$, the problem of finding necessary and sufficient conditions for existence of solutions of

$$
\inf \left\{\int_{\Omega} f(d u(x)) d x: u \in u_{\zeta_{0}}+W_{0}^{1, \infty}\left(\Omega ; \mathbb{R}\left(\begin{array}{c}
n \\
k
\end{array}\right)\right)\right\}
$$

where the boundary data $u_{\zeta_{0}}$ satisfies $d u_{\zeta_{0}}=\zeta_{0}$, for $\zeta_{0}$ a given vector in

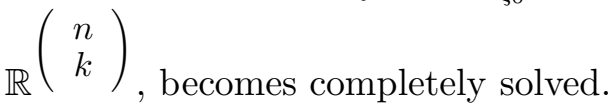

\section{Preliminaries}

We start with some notations which are used throughout this paper. Although these notations are somewhat standard we mention them here for the sake of completeness.

- $\mathbb{R}_{0}^{+}$denotes the set of all non-negative real numbers.

- For $E \subseteq \mathbb{R}^{N}, E \neq \emptyset$, we write span $E$ to denote the subspace spanned by $E$.

- Let $W$ be a subspace of $\mathbb{R}^{N}$. We write $\operatorname{dim} W$ to denote the dimension of $W$.

- $H^{k}$ denotes the $k$-dimensional Hausdorff measure.

- $B(\Omega)$ denotes the Borel $\sigma$-algebra of subsets of $\Omega$.

- co $U$ denotes the convex hull of $U \subseteq \mathbb{R}^{N}$ and $\overline{c o} U$ its closure.

- For a function $f: \mathbb{R}^{N} \rightarrow \mathbb{R}, f^{* *}$ denotes the convex envelope of $f$, that is,

$$
f^{* *}=\inf \{g: g \text { convex, } g \leq f\} .
$$

- $\Omega$ denotes an open bounded subset of $\mathbb{R}^{n}$, and we denote its Lebesgue measure by meas $(\Omega)$.

- We denote by $B_{n}(x, \epsilon)$ the open ball in $\mathbb{R}^{n}$ centered at $x$ with radius $\epsilon$.

- the letter $C$ will be used throughout this work to indicate a constant whose value might change fro line to line.

- We use the standard multi-index notation: for $\alpha=\left(\alpha_{1}, \ldots, \alpha_{n}\right)$, $\alpha_{j} \in \mathbb{N}, j=1, \ldots, n,|\alpha|=\alpha_{1}+\ldots+\alpha_{n}$, and for $u: \mathbb{R}^{n} \rightarrow \mathbb{R}^{N}, \partial^{\alpha} u_{i}$ 
denotes the partial derivative

$$
\partial^{\alpha} u_{i}=\frac{\partial^{|\alpha|} u_{i}}{d x_{1}^{\alpha_{1}} d x_{2}^{\alpha_{2}} \ldots d x_{n}^{\alpha_{n}}} .
$$

\section{Statement of the problem}

For $P D$ as above and for $f: \mathbb{R} \rightarrow \mathbb{R}$ a continuous function, we look for necessary and sufficient conditions for existence of solutions of the following minimization problem:

$$
\inf \left\{\int_{\Omega} f(P D u(x)) d x: u \in u_{\zeta_{0}}+W_{0}^{m, \infty}\left(\Omega ; \mathbb{R}^{N}\right)\right\}
$$

where:

a) for $i=1, \ldots, N, A_{i}=\left\{\alpha, 0<|\alpha| \leq m_{i}\right\}$ is a set of multi-indices, where $m_{i} \in \mathbb{N}_{1}$ and for $u: \mathbb{R}^{n} \rightarrow \mathbb{R}^{N}, P D$ is the linear partial differential operator given by

$$
P D u=\sum_{i=1}^{N} \sum_{\alpha \in A_{i}} c_{\alpha, i} \partial^{\alpha} u_{i},
$$

under one of the following hypothesis:

$$
\left(H_{1}\right) \quad \exists i \in\{1, \ldots, N\}:|\alpha|=m_{i}, \forall \alpha \in A_{i} .
$$

or

$$
\left(H_{2}\right) \quad \exists i \in\{1, \ldots, N\}, \exists j \in\{1 \ldots, n\}: \alpha_{j}=\text { constant, } \forall \alpha \in A_{i} .
$$

b) the boundary data $u_{\zeta_{0}}$ satisfies $P D u_{\zeta_{0}}(x)=\zeta_{0}$, for $\zeta_{0} \in \mathbb{R}$ given, and $m=\max \left\{m_{i}, i=1, \ldots, N\right\}$,

It will be helpfull to consider the auxiliary problem

$$
\left(P^{* *}\right) \quad \inf \left\{\int_{\Omega} f^{* *}(P D u(x)) d x: u \in u_{\zeta_{0}}+W_{0}^{m, \infty}\left(\Omega ; \mathbb{R}^{N}\right)\right\}
$$

where $f^{* *}$ is the convex envelpe of $f$. By convexity of $f^{* *}$, using Jensen's inequality and the density of $C_{c}^{\infty}$ in $W_{0}^{m, \infty}$, it is easy to see that

$$
\inf \left(P^{* *}\right)=f^{* *}\left(\zeta_{0}\right) \operatorname{meas}(\Omega) \text {. }
$$




\section{The Approximation Lemma}

In this section we prove an approximation lemma which will be used to establish suufficient conditions for the existence of solutions of problem $(P)$. This result also allows us to prove a relaxation result which states that $\inf (P)=\inf \left(P^{* *}\right)$.

Lemma 4.1. Let $\Omega \subset \mathbb{R}^{n}$ open, bounded and let $t \in[0,1]$ and $\zeta, \eta \in \mathbb{R}$. Let $\phi \in W^{m, \infty}\left(\Omega ; \mathbb{R}^{N}\right)$ be such that $P D \phi=t \zeta+(1-t) \eta$. Then, for every $\epsilon>0$, there exist $u \in \phi+W_{0}^{m, \infty}\left(\Omega ; \mathbb{R}^{N}\right)$ and $\Omega_{\zeta}, \Omega_{\eta}$, disjoint open subsets of $\Omega$ such that

$$
\left\{\begin{array}{c}
\mid \text { meas }\left(\Omega_{\zeta}\right)-t \text { meas }(\Omega) \mid \leq \epsilon \\
\mid \text { meas }\left(\Omega_{\eta}\right)-(1-t) \operatorname{meas}(\Omega) \mid \leq \epsilon \\
u=\phi \text { in a neighbourhood of } \partial \Omega \\
\|u-\phi\|_{L^{\infty}} \leq C \epsilon \\
P D u(x)=\left\{\begin{array}{l}
\zeta \text { in } \Omega_{\zeta} \\
\eta \text { in } \Omega_{\eta}
\end{array}\right. \\
\operatorname{dist}(P D u(x),[\zeta, \eta]) \leq C \epsilon, \text { a.e. in } \Omega,
\end{array}\right.
$$

where $[\zeta, \eta]$ denotes the closed interval with endpoints $\zeta$ and $\eta$.

Proof. We are assuming that $\zeta \neq \eta$, otherwise the result is trivial since it suffices to take $u=\phi$. We follow here the ideas presented in [2].

Without loss of generality we may assume that $\Omega$ is the unit cube centered at the origin with its faces parallel to the coordinate axes. Indeed, if this is not the case, we can express $\Omega$ as the disjoint union of cubes whose faces are parallel to the coordinate axes plus a set of small measure; in this case a solution $u$ for $(4.1)$ with respect to $\Omega$ can be constructed from a solution of (4.1) when $\Omega$ is the unit cube by setting $u=\phi$ on the set of small measure and by using homothetics and translations in each of the small subcubes.

Let $\epsilon>0$, let $\Omega_{\epsilon} \subset \subset \Omega$ and let $h \in C_{0}^{\infty}(\Omega)$ and $L=L(\Omega)$ be such that 


$$
\left\{\begin{array}{l}
\text { meas }\left(\Omega \backslash \Omega_{\epsilon}\right) \leq \frac{\epsilon}{2} \\
0 \leq h(x) \leq 1, \forall x \in \Omega \\
h(x)=1, \forall x \in \Omega_{\epsilon} \\
\left|D^{k} h(x)\right| \leq L \epsilon^{-k}, \forall x \in \Omega \backslash \Omega_{\epsilon}, \forall 0<k \leq m
\end{array}\right.
$$

Let $\delta>0$. Suppose first that $P D$ satisfies $\left(H_{1}\right)$ and that $N=1$. Let $A$ be the set of all multi-indices interveening in $P D$, and let $\overline{\alpha_{1}}=\max \left\{\alpha_{1}, \alpha \in\right.$ $A$ \}. Select one $\bar{\alpha} \in A$ such that its first component equals $\overline{\alpha_{1}}$ (there could be more than one). Notice that w.l.o.g. we are assuming that $\overline{\alpha_{1}}>0$ (otherwise, take other variable involved). By a standard procedure we may construct a $C^{\infty}$ function $g:[0,1] \rightarrow \mathbb{R}$ and sets $I_{\zeta}, I_{\eta}$ which are unions of dispoint open subintervals of $[0,1]$, so that

$$
\left\{\begin{array}{l}
g^{\overline{\alpha_{1}}}\left(x_{1}\right)=\left\{\begin{array}{l}
1-t \quad \text { if } x_{1} \in I_{\zeta} \\
-t \quad \text { if } x_{1} \in I_{\eta}
\end{array}\right. \\
g^{\overline{\alpha_{1}}}\left(x_{1}\right) \in[-t, 1-t], \forall x_{1} \in[0,1], \\
\left|\operatorname{meas}\left(I_{\zeta}\right)-t\right| \leq \frac{\epsilon}{2}, \\
\left|\operatorname{meas}\left(I_{\eta}\right)-(1-t)\right| \leq \frac{\epsilon}{2}, \\
\left.\left|g\left(x_{1}\right)\right| \leq \delta, \mid g^{\prime}\left(x_{1}\right)\right) \leq \delta, \ldots,\left|g^{\overline{\alpha_{1}}-1}\left(x_{1}\right)\right| \leq \delta \forall x_{1} \in[0,1]
\end{array}\right.
$$

Let

$$
\Omega_{\zeta}:=\left\{x \in \Omega_{\epsilon}: x_{1} \in I_{\zeta}\right\}
$$

and

$$
\Omega_{\eta}:=\left\{x \in \Omega_{\epsilon}: x_{1} \in I_{\eta}\right\}, .
$$

We now define the function $w \in C^{\infty}(\bar{\Omega})$ by

$$
w(x)=\frac{1}{C_{\bar{\alpha}}\left(\alpha_{2} ! \ldots \alpha_{n} !\right)} g\left(x_{1}\right) x_{2}^{\overline{\alpha_{2}}} \ldots x_{n}^{\overline{\alpha_{n}}}(\zeta-\eta) .
$$

If $u: \Omega \subset \mathbb{R}^{n} \rightarrow \mathbb{R}^{N}, N>1$, the same process works with the necessary adaptations. In fact, we just need to construct $w_{1}$ as before (i.e. like $w$ in the case $N=1$, regarding the component $u_{1}$ of $u$ ) and then set;

$$
w=\left(w_{1}, 0, \ldots, 0\right) \text {. }
$$


(where, once again, we assumed w.l.o.g. that the component $u_{1}$ is involved by the operator $P D$.)

We claim that the function $u: \Omega \rightarrow \mathbb{R}$ given by

$$
u=\phi+h w
$$

satisfies the properties listed in (4.1). Indeed, since $h \equiv 1$ in $\Omega_{\epsilon}$, we have

$$
\begin{aligned}
P D(u) & =P D \phi+P D(h w) \\
& =t \zeta+(1-t) \eta+g^{\overline{\alpha_{1}}}\left(x_{1}\right)(\zeta-\eta)
\end{aligned}
$$

so that

$$
P D u(x)= \begin{cases}\zeta & \text { in } \Omega_{\zeta} \\ \eta & \text { in } \Omega_{\eta}\end{cases}
$$

Using the fact that $h \leq 1$ and $g\left(x_{1}\right) \mid \leq \delta$, it is possible to choose $\delta$ sufficiently small with respect to $\epsilon$ so that $\|u-\phi\|_{\infty}=\|h w\|_{\infty}<\epsilon$.

On he other hand,

$$
\begin{aligned}
P D u & =P D \phi+P D(h w) \\
& =t \zeta+(1-t) \eta+h g^{\overline{\alpha_{1}}}\left(x_{1}\right)(\zeta-\eta)+R(h, \alpha) \\
& =\left(t+h g^{\overline{\alpha_{1}}}\left(x_{1}\right)\right) \zeta+\left(1-\left(t+h g^{\overline{\alpha_{1}}}\left(x_{1}\right)\right)\right) \eta+R(h, \alpha),
\end{aligned}
$$

where, using the estimates on the derivatives of $h$ and $g$, we can once again choose $\delta$ sufficiently small with respect to $\epsilon$, in order to obtain

$$
|R(h, \alpha)| \leq \epsilon .
$$

Since $0 \leq t+h g^{\overline{\alpha_{1}}}\left(x_{1}\right) \leq 1$, by $(4.2)$ and (4.3) we conclude that

$$
\operatorname{dist}(P D u(x),[\zeta, \eta]) \leq|R(h, \alpha)| \leq \epsilon \text {, a.e. in } \Omega \text {. }
$$

The proof of the remaining statements $f(4.1)$ is straightforward.

Suppose now that $P D$ satisifes $\left(H_{2}\right)$. The process is similar, just constructing $w$ using one of the lowest order derivatives.

Theorem 4.1. Let $f: \mathbb{R} \rightarrow \mathbb{R}$ continuous. Then

$$
\inf (P)=\inf \left(P^{* *}\right) .
$$

Proof. The inequality $\inf (P) \geq \inf \left(P^{* *}\right)$ is trivial. We will use the fact that $\inf \left(P^{* *}\right)=f^{* *}\left(\zeta_{0}\right)$ meas $(\Omega)$ to prove the reverse inequality. 
Given $\zeta_{0} \in \mathbb{R}$ there exist $t_{i}>0$ and $\zeta_{i} \in \mathbb{R}$ such that $\sum_{i=1}^{2} t_{i}=1, \zeta_{0}=$ $\sum_{i=1}^{2} t_{i} \zeta_{i}$ and

$$
f^{* *}\left(\zeta_{0}\right)=\sum_{i=1}^{2} t_{i} f\left(\zeta_{i}\right)
$$

Applying the previous lemma to $\zeta_{0}$ and $u_{\zeta_{0}}$ we obtain a sequence $u_{n} \in$ $u_{\zeta_{0}}+W_{0}^{m, \infty}(\Omega)$, and $\Omega_{i}, i=1,2$, disjoint open subsets of $\Omega$ such that

$$
\left\{\begin{array}{l}
\mid \text { meas }\left(\Omega_{i}\right)-t_{i} \operatorname{meas}(\Omega) \mid \leq \frac{1}{n}, i=1,2, \\
\left\|u_{n}-u_{\zeta_{0}}\right\|_{\infty} \leq \frac{C}{n} \\
P D u_{n}(x)=\zeta_{i} \text { in } \Omega_{i}, i=1,2 \\
\operatorname{dist}\left(P D u_{n}(x), \operatorname{co}\left\{\zeta_{1}, \zeta_{2}\right\}\right) \leq \frac{C}{n}, \text { a.e. in } \Omega .
\end{array}\right.
$$

The sequence $u_{n}$ is admissible for problem $(P)$, so we have

$$
\begin{aligned}
& \inf (P) \leq \lim _{n \rightarrow \infty} \int_{\Omega} f\left(P D u_{n}(x)\right) d x \\
& =\lim _{n \rightarrow \infty}\left(\int_{\Omega \backslash \cup_{i=1}^{2} \Omega_{i}} f\left(P D u_{n}(x)\right) d x+\sum_{i=1}^{2} \int_{\Omega_{i}} f\left(\zeta_{i}\right) d x\right) \\
& \leq \lim _{n \rightarrow \infty}\left(\int_{\Omega \backslash \cup_{i=1}^{2} \Omega_{i}} f\left(P D u_{n}(x)\right) d x+\sum_{i=1}^{2} f\left(\zeta_{i}\right) t_{i} \operatorname{meas}(\Omega)+\frac{C}{n}\right) \\
& =f^{* *}\left(\zeta_{0}\right) \operatorname{meas}(\Omega)=\inf \left(P^{* *}\right),
\end{aligned}
$$

where

$$
\lim _{n \rightarrow \infty} \int_{\Omega \backslash \cup_{i=1}^{2} \Omega_{i}} f\left(P D u_{n}(x)\right) d x=0
$$

since meas $\left(\Omega \backslash \cup_{i=1}^{2} \Omega_{i}\right) \leq \frac{2}{n}$ and by the continuity of $f$, the sequence $\left(f\left(P D u_{n}().\right)\right)$ is uniformly bounded.

\section{Necessary Conditions}

We look for necessary conditions for the existence of solutions of the problem

$$
(P) \quad \inf \left\{\int_{\Omega} f(P D u(x)) d x: u \in u_{\zeta_{0}}+W^{m, \infty}\left(\Omega ; \mathbb{R}^{N}\right)\right\}
$$

where the boundary data $u_{\zeta_{0}}$ satisfies $P D U_{\zeta_{0}}(x)=\zeta_{0}$, for $\zeta_{0} \in \mathbb{R}$ given and $f: \mathbb{R} \rightarrow \mathbb{R}$ is continuous. 
The set

$$
K:=\left\{\zeta \in \mathbb{R}: f^{* *}(\zeta)<f(\zeta)\right\}
$$

will play an important role in this analysis.

Proposition 5.1. Suppose $u$ solves $(P)$. Then $u$ is a solution of

$$
\begin{cases}f(P D u)=f^{* *}(P D u) & \text { a.e. in } \Omega \\ u=u_{\zeta_{0}} & \text { on } \partial \Omega\end{cases}
$$

Proof. If $u$ is a solution of $(P)$ then clearly $u=u_{\zeta_{0}}$ on $\partial \Omega$. On the other hand, by Theorem (4.1), we know that

$$
\inf (P)=\inf \left(P^{* *}\right)=f^{* *}\left(\zeta_{0}\right) \operatorname{meas}(\Omega) .
$$

Hence, by convexity of $f^{* *}$, Jensen's inequality and by density, we have that

$$
\begin{aligned}
& f^{* *}\left(\zeta_{0}\right) \operatorname{meas}(\Omega)=\inf (P)=\int_{\Omega} f(P D u(x)) d x \\
& \geq \int_{\Omega} f^{* *}\left(P D u_{n}(x)\right) d x \geq f^{* *}\left(\frac{1}{\operatorname{meas}(\Omega)} \int_{\Omega} P D u(x) d x\right) \operatorname{meas}(\Omega) \\
& =f^{* *}\left(\frac{1}{\operatorname{meas}(\Omega)} \int_{\Omega} \zeta_{0}+P D\left(u(x)-u_{\zeta_{0}}(x)\right) d x\right) \operatorname{meas}(\Omega) \\
& =f^{* *}\left(\zeta_{0}\right) \operatorname{meas}(\Omega) .
\end{aligned}
$$

It follows that $f(P D U(x))=f^{* *}(P D u(x))$ for a.e. $x \in \Omega$.

Remark 5.1. When $\zeta_{0}$ is a vector (for instance in the case of the gradient or the curl) there is another necessary condition related to the existence of directions of strict convexity for $f^{* *}$ at $\zeta_{0}$. However, in this simpler case, $f^{* *}$ is affine in each connected component of $K$.

\section{Sufficient Conditions}

\section{Recall that}

$$
K=\left\{\zeta \in \mathbb{R}: f^{* *}(\zeta)<f(\zeta)\right\},
$$

and that the boundary data $u_{\zeta_{0}}$ satisfies $P D u_{\zeta_{0}}=\zeta_{0}$. Our main result in this section is the following

Theorem 6.1. Let $K \subset \mathbb{R}$ be bounded and connected and let $\zeta_{0} \in K$. Then, if $u \in W^{m, \infty}\left(\Omega ; \mathbb{R}^{N}\right)$ is a solution of

$$
\left(I_{1}\right) \quad\left\{\begin{array}{c}
P D u \in \partial K \\
u=u_{\zeta_{0}} \text { on } \partial \Omega
\end{array}\right.
$$

then $u$ is also a solution of $(P)$. 
Proof. Since $f^{* *}$ is affine in $\bar{K}$ at $\zeta_{0}$, if $u$ is a solution of $\left(I_{1}\right)$, then there exist $a, b \in \mathbb{R}$, such that $f^{* *}(P D u)=a P D u+b$. As $P D u \in \partial K$, we have that

$$
\begin{aligned}
\int_{\Omega} f(P D u(x)) d x & =\int_{\Omega} f^{* *}(P D u(x)) d x \\
& =\int_{\Omega} a P D u(x)+b d x
\end{aligned}
$$

Since

$$
\int_{\Omega} a\left(u(x)-u_{\zeta_{0}}(x)\right) d x=0
$$

and hence

$$
\int_{\Omega} a P D u(x) d x=\int_{\Omega} a \zeta_{0} d x
$$

we conclude that

$$
\begin{aligned}
\int_{\Omega} f(P D u(x)) d x & =\int_{\Omega} a \zeta_{0}+b d x \\
& =f^{* *}\left(\zeta_{0}\right) \operatorname{meas}(\Omega) \\
& =\inf \left(P^{* *}\right)=\inf (P)
\end{aligned}
$$

that is, $u$ is a non-trivial solution of $(P)$. Notice that, since $\zeta_{0} \in \partial K, u_{\zeta_{0}}$ is not a solution of $\left(I_{1}\right)$, and so, by Proposition (4.1), it is not a solution of $(P)$ either. If $f^{* *}$ is globally affine the equalities (6.4) hold trivially and so the result follows as in the first case.

Before proving existence of solutions to problem $\left(I_{1}\right)$, we need the following definitions:

Definition 6.1. Let $\Omega \subseteq \mathbb{R}^{n}$ be an open set. For $\theta>0$, let $W_{\theta}$ be the set of all functions $u \in C_{\text {piec }}^{m}\left(\bar{\Omega} ; \mathbb{R}^{N}\right)$ for which there exists $\Omega_{\theta} \subset \Omega$ such that meas $\left(\Omega \backslash \Omega_{\theta}\right) \leq \theta$, and $P D u$ is piecewise constant in $\Omega_{\theta}$.

Definition 6.2. Let $E ; S \subseteq \mathbb{R}$. We say that $S$ has the relaxation property with respect to $E$, if, for every bounded open set $\Omega \subseteq \mathbb{R}^{n}$ and for every map $u_{\zeta}$ satisfying $P D u_{\zeta}=\zeta \in \operatorname{int} S$, there exists a sequence $u: n \in W_{\frac{1}{n}}$ such 
that

$$
\left\{\begin{array}{l}
u_{n} \in u_{\zeta}+W_{0}^{m, \infty}\left(\Omega ; \mathbb{R}^{N}\right) \\
u_{n} \rightarrow u_{\zeta} \text { in } L^{\infty}\left(\Omega ; \mathbb{R}^{N}\right), P D u_{n} \stackrel{*}{\rightarrow} \zeta \text { in } L^{\infty}(\Omega) \\
P D u_{n}(x) \in E \cup \operatorname{int} S \text { a.e. in } \Omega \\
\lim _{n \rightarrow+\infty} \int_{\Omega} \operatorname{dist}\left(P D u_{n}(x), E\right) d x=0
\end{array}\right.
$$

For the proof of existence of solutions to problem $\left(I_{1}\right)$, we will need to show that $\operatorname{co}(\partial K)$ has the relaxation property with respect to $\partial K$. The following lemma provides a way of doing this.

Lemma 6.1. Let $E, E_{\delta} \subseteq \mathbb{R}$, for $\left.\delta \in\right] 0, \delta_{0}[$, be compact sets such that

i) $\operatorname{co} E_{\delta} \subset$ intco $E$ for every $\left.\delta \in\right] 0, \delta_{0}[$;

ii) $\forall \epsilon>0, \exists \delta(\epsilon)>0$, such that, $\forall \delta \in] 0, \delta(\epsilon)\left[, \rho \in E_{\delta} \Rightarrow \operatorname{dist}(\rho, E) \leq \epsilon\right.$;

iii) if $\rho \in \operatorname{intco} E$, then $\rho \in \operatorname{co} E_{\delta}$, for every $\delta>0$ sufficiently small.

Then $\operatorname{co} E$ has the relaxation property with respect to $E$.

Proof. Let $\Omega \subset \mathbb{R}^{n}$ be a bounded open set and let $u: \Omega \rightarrow \mathbb{R}^{N}$ be a function satisfying

$$
P D u=\zeta \in \operatorname{intco} E .
$$

We claim that there exists a sequence $u_{n} \in W_{\frac{1}{n}}$ such that

$$
\left\{\begin{array}{l}
u_{n} \in u+W_{0}^{m, \infty}\left(\Omega ; \mathbb{R}^{N}\right) \\
u_{n} \rightarrow u \text { in } L^{\infty}\left(\Omega ; \mathbb{R}^{N}\right), P D u_{n} \stackrel{*}{ } \zeta \text { in } L^{\infty}(\Omega) \\
P D u_{n}(x) \in E \cup \operatorname{intco} E \text { a.e. in } \Omega \\
\lim _{n \rightarrow+\infty} \int_{\Omega} \operatorname{dist}\left(P D u_{n}(x), E\right) d x=0
\end{array}\right.
$$

Fix $\epsilon>0$ and let $\delta=\delta(\epsilon)$ be determined according to $i i$ ). By iii), we may find $\delta_{1}<\delta$ such that

$$
\zeta \in \operatorname{intco} E \Rightarrow \zeta \in \operatorname{co} E_{\delta_{1}}
$$

Therefore we may write $\zeta=t \zeta_{1}+(1-t) \zeta_{2}$ with $\zeta_{1}, \zeta_{2} \in E_{\delta_{1}}$. By property $i$ ), we can choose $\left.\epsilon^{\prime} \in\right] 0, \epsilon\left[\right.$ such that the $\epsilon^{\prime}$-neighbourhood of $\operatorname{co} E_{\delta_{1}}$ is contained in intco $E$. Using the Approximation Lemma (4.1) we may find 
$u_{\epsilon} \in C_{\text {piec }}^{m}\left(\bar{\Omega} ; \mathbb{R}^{N}\right)$ and $\Omega_{1}, \Omega_{2}$ disjoint open subsets of $\Omega$, such that

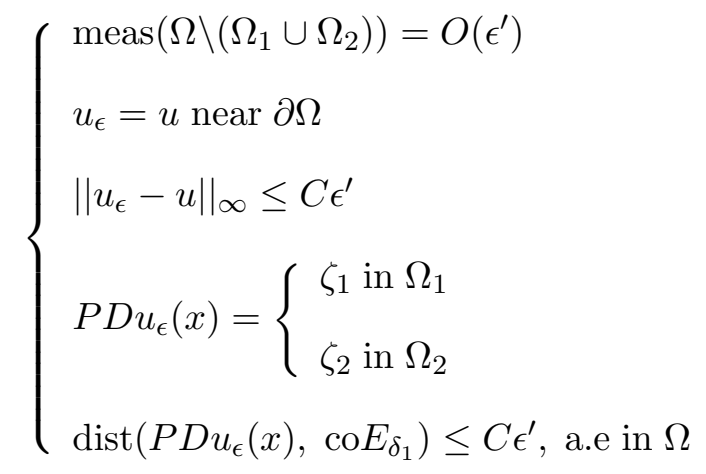

Now, the choice of $\epsilon^{\prime}$ ensures that

$$
P D u_{\epsilon} \in \operatorname{co} E,
$$

and in view of $i i)$, taking into account that $\operatorname{dist}\left(P D u_{\epsilon}, E\right)$ is a bounded function, we conclude that

$$
\begin{aligned}
\int_{\Omega} \operatorname{dist}\left(P D u_{\epsilon}(x), E\right) d x & =\int_{\Omega_{1}} \operatorname{dist}\left(\zeta_{1}, E\right) d x+\int_{\Omega_{2}} \operatorname{dist}\left(\zeta_{2}, E\right) d x \\
& +\int_{\Omega \backslash\left(\Omega_{1} \cup \Omega_{2}\right)} \operatorname{dist}\left(P D u_{\epsilon}(x) d x\right. \\
& \leq \epsilon \operatorname{meas}\left(\Omega_{1}\right)+\epsilon \operatorname{meas}\left(\Omega_{2}\right)+O\left(\epsilon^{\prime}\right)=O(\epsilon)
\end{aligned}
$$

so that the claim is obtained by letting $\epsilon \rightarrow 0^{+}$. Finally, we need to check if $u_{n} \in W_{\frac{1}{n}}$. Letting $2 \epsilon^{\prime}=\frac{1}{n}$, we have that $\left(u_{n}\right) \subset C_{\text {piec }}^{m}\left(\bar{\Omega} ; \mathbb{R}^{N}\right)$ and

$$
P D u_{n}(x)=\left\{\begin{array}{l}
\zeta_{1} \text { in } \Omega_{1} \\
\zeta_{2} \text { in } \Omega_{2}
\end{array}\right.
$$

where meas $\left(\Omega \backslash\left(\Omega_{1} \cup \Omega_{2}\right)\right) \leq \frac{1}{n}$. Thus, $u_{n} \in W_{\frac{1}{n}}$.

In what follows, we will need the following result of convex analysis, which can be found in [11].

Proposition 6.1. Let $E \subset \mathbb{R}$ be compact and such that intco $E \neq \emptyset$. Let $E_{\text {ext }}$ denote the set of extreme points of $\mathrm{co} E$. Then, there exists a convex a lower semicontinuous function $\psi: \mathbb{R} \rightarrow \mathbb{R} \cup\{+\infty\}$ such that

$$
E_{\text {ext }}=\{\zeta \in \mathbb{R}: \psi(\zeta)=0\}
$$

and

$$
\operatorname{co} E=\operatorname{co} E_{\text {ext }}=\{\zeta \in \mathbb{R}: \psi(\zeta) \leq 0\} .
$$

Finally we are in position to state the following 
Theorem 6.2. Let $\Omega \subseteq \mathbb{R}^{n}$ be an open set and let $E \subset \mathbb{R}$ be a compact set. Let $\phi \in C_{\text {piec }}^{m}\left(\bar{\Omega} ; \mathbb{R}^{\bar{N}}\right)$ be such that $P D \phi$ is piecewise constant in $\Omega$ an $P D \phi \in E \cup$ intco $E$. Then there exists $u \in C\left(\bar{\Omega} ; \mathbb{R}^{N}\right)$ with $P D u \in L^{\infty}(\Omega)$ and satisfying

$$
\left\{\begin{array}{c}
P D u(x) \in E \text { a.e. } x \in \Omega \\
u(x)=\phi(x), \forall x \in \partial \Omega
\end{array}\right.
$$

Proof. Assume w.l.o.g. that $\Omega$ is bounded and that $\left.\phi \in C^{m}(\bar{\Omega}) ; \mathbb{R}^{N}\right)$. Assume also that intco $E \neq \emptyset$ since otherwise the result is trivial (it suffices to take $u \equiv \phi)$.

\section{Step 1}

Under the previous assumptions we show that $\operatorname{co} E$ has the relaxation property with respect to $E$. For this purpose we use Lemma (6.1). Choose $\alpha_{0} \in$ intco $E$ and, for $\left.\delta \in\right] 0,1[$, define the sets

$$
E_{\delta}:=\delta \alpha_{0}+(1-\delta) E .
$$

Notice that these sets are compact since $E$ is compact. If $\rho \in E_{\delta}$ then $\rho=\delta \alpha_{0}+(1-\delta) \bar{\rho}$, with $\bar{\rho} \in E$, and hence

$$
\operatorname{dist}(\rho, E) \leq|\rho-\bar{\rho}|<\epsilon,
$$

provided we take $\delta(\epsilon)=\frac{\epsilon}{\operatorname{diamcoE}}$. This proves property $\left.i i\right)$. As for property iii), if $\rho \in \operatorname{intco} E$, since

$$
\lim _{\delta \rightarrow 0}\left|\left(\frac{\rho}{1-\delta}-\frac{\delta}{1-\delta} \alpha_{0}\right)-\rho\right|=0
$$

it follows that

$$
\frac{\rho}{1-\delta}-\frac{\delta}{1-\delta} \alpha_{0} \in \operatorname{co} E
$$

for $\delta>0$ sufficiently small, and so $\rho \in \delta \alpha_{0}+(1-\delta) \operatorname{co} E=\operatorname{co} E_{\delta}$.

It remains to show property $i$ ). Since $\alpha_{0} \in \operatorname{intco} E$ there exists $r>0$ such that

$$
B_{1}\left(\alpha_{0}, r\right) \subset \operatorname{co} E .
$$

Therefore, it suffices to show that

$$
B_{1}\left(\delta \alpha_{0}+(1-\delta) \rho, \delta r\right) \subset \operatorname{co} E, \forall \rho \in \operatorname{co} E .
$$

Let $x \in B_{1}\left(\delta \alpha_{0}+(1-\delta) \rho, \delta r\right)$ and let

$$
\alpha:=\frac{x-(1-\delta) \rho}{\delta} .
$$


Notice that $\left|\delta \alpha-\delta \alpha_{0}\right|<\delta r$ and so, from (6.5), we have that $\alpha \in \operatorname{co} E$, and we conclude that

$$
x=\delta \alpha+(1-\delta) \rho \in \operatorname{co} E .
$$

\section{Step 2}

Assume first that $\operatorname{PD} \phi(x) \in$ intco $E$ for a.e. $x \in \Omega$. Since $E$ is compact and intco $E \neq \emptyset$, by Proposition (6.1) applied to $E_{\text {ext }}$, we conclude that there exists a convex and lower semicontinuous function

$$
\psi: \mathbb{R} \rightarrow \mathbb{R} \cup\{+\infty\},
$$

such that

and

$$
E=E_{\text {ext }}=\{\zeta \in \mathbb{R}: \psi(\zeta)=0\}
$$

$$
\operatorname{co} E=\operatorname{co} E_{\text {ext }}=\{\zeta \in \mathbb{R}: \psi(\zeta) \leq 0\}
$$

Let $V$ be the set of fuctions $u: \Omega \rightarrow \mathbb{R}^{N}$ so that there exists $u_{n} \in W_{\frac{1}{n}}$ satisfying $u_{n}=\phi$ on $\partial \Omega, P D u_{n} \in E \cup$ intco $E$ a.e. in $\Omega$, and $u_{n} \rightarrow u$ in $L^{\infty}\left(\Omega ; \mathbb{R}^{N}\right)$. Notice that $\phi \in V$ and $V$ is a complete metric space when endowed with the $C^{0}$ norm.

The compactness of $E$ and $\operatorname{co} E$, the convexity and lower semicontinuity of $\psi$ and (6.6), (6.7) yield

$$
\begin{aligned}
& V \subset\left\{u \in C\left(\bar{\Omega} ; \mathbb{R}^{N}\right): P D u \in L^{\infty}(\Omega),\right. \\
& u=\phi \text { on } \partial \Omega, \psi(P D u(x)) \leq 0 \text { a.e. } x \in \Omega\} .
\end{aligned}
$$

For $u \in V$, set

$$
L(u):=\int_{\Omega} \psi(P D u(x)) d x .
$$

From the lower semicontinuity and convexity of $\psi$ we have that, for every $u \in V$,

$$
L(u) \leq \liminf _{u_{n} \rightarrow, u_{n} \in V} L\left(u_{n}\right) .
$$

Also, by (6.8), $L(u) \leq 0$ and

$$
L(u)=0 \Leftrightarrow P D u(x) \in E \text { for a.e. } x \in \Omega .
$$

Define

$$
V^{k}:=\left\{u \in V: L(u)>-\frac{1}{k}\right\} .
$$

By (6.9), $V^{k}$ is open in $V$. We will now prove that $V^{k}$ is dense in $V$, in which case it will follow from Baire's Category Theorem that $\bigcap_{k} V^{k}$ is dense in $v$. In particular,

$$
\bigcap_{k} V^{k}=\{u \in V: L(u)=0\} \neq \emptyset .
$$


Thus, there exists $u \in V$ such that $L(u)=0$, that is, by (6.10), such that $P D u(x) \in E=E_{\text {ext }}$ for a.e. $x \in \Omega$, and since $u \in V$ we are done.

Therefore, it remains to prove the density result, i.e., that for fixed $k \in$ $\mathbb{N}, u \in V$, and $\epsilon \in] 0, \frac{1}{k}$ [ sufficiently small, we can find $u_{\epsilon} \in V^{k}$ such that $\left\|u_{\epsilon}-u\right\|_{\infty} \leq \epsilon$. We will prove this property under the further assumption that, for some $\theta>0$, small, $u \in W_{\theta}$ and $P D u(x) \in E \cap$ intco $E$ for a.e. $x \in$ $\Omega$. The general case will follow from the definition of $V$. Also, by working on each subset of $\Omega$ where $u$ is of class $C^{m}$ and $P D u$ is constant, and by setting $u_{\epsilon}=u$ on $\Omega \backslash \Omega_{\theta}$ we can assume, w.l.o.g., that $u \in C^{m}\left(\bar{\Omega} ; \mathbb{R}^{N}\right), P D u$ is constant in $\Omega$ and $P D u(x) \in$ intco $E$ (otherwise the result is trivial).

By compactness of $E$ and $\operatorname{co} E$ we have that

$$
\zeta \in E \cup \operatorname{co} E \Rightarrow \operatorname{dist}(\zeta, E) \leq \beta
$$

for some $\beta>0$. By the convexity and lower semicontinuity of $\psi$ and (6.6), we can fix $\delta=\delta(\epsilon)>0$, such that, for any measurable function $\mu: \mathbb{R}^{n} \rightarrow E \cap \operatorname{co} E$, the following holds

$$
\int_{\Omega} \operatorname{dist}(\mu(x), E) d x \leq \delta \Rightarrow \int_{\Omega} \psi(\mu(x)) d x \geq-\epsilon .
$$

The result now follows immediately from the relaxation property. Indeed, since $\operatorname{co} E$ has the relaxation property with respect to $E$ and since $P D u(x)$ is a constant belonging to intco $E$, there exists a sequence $u_{\epsilon} \in W_{\epsilon}$ such that

$$
\left\{\begin{array}{c}
u_{\epsilon} \in u+W_{0}^{m, \infty}\left(\Omega ; \mathbb{R}^{N}\right) \\
\left\|u_{\epsilon}-u\right\|_{\infty} \leq \epsilon \\
\operatorname{curl} u_{\epsilon}(x) \in E \cup \text { intco } E \text { a.e. } x \in \Omega \\
\int_{\Omega} \operatorname{dist}\left(P D u_{\epsilon}(x), E\right) d x \leq \delta
\end{array}\right.
$$

From (6.12) we conclude that

$$
\int_{\Omega} \psi\left(P D u_{\epsilon}(x)\right) d x \geq-\epsilon \Rightarrow L\left(u_{\epsilon} \geq-\epsilon>-\frac{1}{k}\right.
$$

for $\epsilon<\frac{1}{k}$. Thus $u_{\epsilon} \in V^{k}$ and ||$u_{\epsilon}-\left.u\right|_{-} \infty \leq \epsilon$, so the proof of the density result is complete.

\section{Step 3}

We now turn to the general case, $P D \phi(x) \in E \cup \operatorname{intco} E$ for a.e. $x \in \Omega$, and we let

$$
\Omega_{0}:=\{x \in \Omega: P D \phi(x) \in E\}, \Omega_{1}:=\Omega \backslash \Omega_{0} .
$$


Then $\Omega_{1}$ is open by continuity, and $P D \phi(x) \in \operatorname{intco} E$ for a.e. $x \in \Omega_{1}$. We apply Step 2 to the set $\Omega_{1}$ to obtain a function $u_{1} \in C\left(\bar{\Omega}_{1} ; \mathbb{R}^{N}\right)$ with $P D u_{1} \in L^{\infty}\left(\Omega_{1}\right)$ and such that

$$
\left\{\begin{array}{c}
P D u_{1}(x) \in E_{\text {ext }} \text { for a.e. } x \in \Omega_{1} \\
u_{1}(x)=\phi(x) \text { on } \partial \Omega_{1}
\end{array}\right.
$$

Defining

$$
u(x):= \begin{cases}\phi(x) & \text { if } x \in \Omega_{o} \\ u_{1}(x) & \text { if } x \in \Omega_{1},\end{cases}
$$

it is clear that we find a function satisfying the statement of the theorem.

Corollary 6.1. assume that the set $K$ is bounded and connected and that $\zeta_{0} \in K$. Then there exists $u \in u_{\zeta_{0}}+W_{0}^{m, \infty}\left(\Omega ; \mathbb{R}^{N}\right)$ such that $u$ is a solution of $\left(I_{1}\right)$.

Proof. Set $\phi(x)=u_{\zeta_{0}}(x)$, and $E=\partial K$. Since $K$ is bounded and connected it follows thatt $E$ is compact and that $K \subset \operatorname{co} \partial K$. Thus,

$$
K \subseteq E \cup \operatorname{intco} E
$$

and so $\zeta_{0} \in E \cup$ intco $E$. The existence of a solution to problem $\left(I_{1}\right)$ follows immediately from Theorem (6.2).

\section{References}

[1] S. Bandyopadhyay, B. Dacorogna, A.C. Barroso, J. Matias: Differential inclusions for differential forms, Calc. Var. Partial Differential Equations 28 (2007) 449-469

[2] A.C. Barroso, J. Matias: Necessary and sufficient conditions for existence of solutions of a variational problem involving the curl, Discrete and Continuous Dynamical Systems 12 no. 1 (2005) 97-114.

[3] A. Cellina: On minima of a functional of the gradient: necessary conditions, Nonlinear Analysis, Theory Meth. Appl. 20 (1993) 337-341.

[4] A. Cellina: On minima of a functional of the gradient: sufficient conditions, Nonlinear Analysis, Theory Meth. Appl. 20 (1993) 343-347.

[5] B. Dacorogna: Non convex problems of the calculus of variations and differential inclusions. To appear in Stationary Partial Differential Equations, Handbook of Differential Equations. North-Holland Publishing Co., Amsterdam.

[6] B. Dacorogna: Direct Methods in the Calculus of Variations, Springer-Verlag, Berlin et al.,1989.

[7] B. Dacorogna, I. Fonseca: A-B quasiconvexity and implicit partial differential equations, Calc. Var. Partial Differential Equations 14 no.2 (2002) 115-149.

[8] B. Dacorogna, P. Marcellini: Existence of minimizers for non quasiconvex integrals, Arch. Rat. Mech. Anal. 131 (1995) 359-399.

[9] B. Dacorogna, P. Marcellini: Implicit Partial Differential Equations, Birkhäuser Verlag, Basel,1999. 
[10] I. Fonseca, S. Müller: A-quasiconvexity: a necessary and sufficient condition for lower semicontinuity under PDE constraints, SIAM J. Math. Anal. 30 (1999) $1355-1390$

[11] R. T. Rockafellar: Convex Analysis, Princeton University Press,1970. 